\title{
Dual Band Filter-Antenna with Fixed Low Band and Frequency- Agile High Band for Wireless Communications
}

\author{
Hany A. Atallah \\ Electrical Engineering Department, Faculty of Engineering, South Valley University, Qena 83523, Egypt \\ *corresponding author, E-mail: h. atallah@eng. svu.edu.eg
}

\begin{abstract}
This work proposes a design of compact filter-antenna with fixed and frequency-agile bands for wireless communications and cognitive radio (CR) systems. The filter-antenna is realized by attaching a bandpass filter with an independent fixed band and a tunable band to a UWB antenna. The dual bandpass filter consists of a folded open loop resonator loaded with single varactor diode at a suitable location to achieve reconfigurability operations for the high band while the low band remains fixed at $4.5 \mathrm{GHz}$. A reconfigurable broadband frequency range of $3.2 \mathrm{GHz}$ from 7.8 to $4.6 \mathrm{GHz}$ is achieved for the high band by changing the capacitance of the varactor from 0.16 to $2.25 \mathrm{pF}$, respectively. A prototype of the filter-antenna is fabricated and measured at a selected capacitance of $0.6 \mathrm{pF}$ to verify the simulation results. Good agreements between simulated and measured results are obtained.
\end{abstract}

\section{Introduction}

Recent advancements in wireless communication systems and the growing request for frequency bands have caused congestions in the available electromagnetic (EM) radio frequency (RF) spectrum. Therefore, accessibility of various unlicensed bands in the crowded RF spectrum and compatibility of wireless devices with different standards represent the main objectives of current communication systems to operate with dual frequency bands [1], [2].

In cognitive radio (CR) and ultrawideband (UWB) communication systems [3], one of the keys issues is designing a compact antenna while providing wideband characteristics over the entire operating band and should have a wide reconfigurable band for CR applications [4].

In recent days, the RF front ends and antennas should be designed to operate at multiple frequency bands with frequency-agile characteristics to meet the growing demands and challenges in modern wireless systems and $\mathrm{CR}$ applications [4], [5], [6]. Electronic reconfigurability features are one of the most promising techniques to achieve the frequency-agile characteristics of the antennas [7] and filters [8] and represent a potential solution for future reconfigurable wireless communication systems. Therefore, there is an essential demand for designing reconfigurable antennas that can support the communication system operating with other devices through multi-channels. Also, achieving wide tuning ranges with steady radiation patterns are challenging features because of the matching problems and the switching devices limitations. In these systems, printed slot antennas have gained significant consideration due to their wideband matching characteristics and omnidirectional radiation patterns [9], [10]. However, they suffer from matching problems when converted to single tunable band operations with wide tunability range. The rapid development in designing antennas has produced many different techniques for realizing multi-band operations, compact, low priced, flexible, broadband frequency-Agile, and multiple bands antennas [11], [12].

Lately, reconfigurability for multi-band antennas has gained noteworthy devotion. Several applied approaches for realizing dual band antennas employ specific methodologies to alter one of the resonant modes of the structure to vary its operating frequency. For example, in [13], a $50 \times 50 \mathrm{~mm}^{2}$ dual reconfigurable antenna is achieved using a mechanical process for a movable superstrate. However, the system suffers from the low switching speed of the mechanical part. In [14], a multi-band antenna is proposed by using a fundamental patch and four sub-patches to produce five resonant frequencies. The sub-patches are loaded at the inputs with varactors for reconfiguration operations. The antenna has a size of $50 \times 50 \mathrm{~mm}^{2}$. In [8], a dual band bandpass filter with fixed low-band and reconfigurable high-band resonant frequencies is suggested for reconfigurable applications. The suggested filter contains double split-ring resonators (SRRs) with stub and microfluidic channels. The tunability of the high resonant frequency is achieved by controlling the stub length by injecting liquid metal in the microfluidic channel. The filter has a size of $80 \times 30 \mathrm{~mm}^{2}$. However, the injection process needs cautious treatments. In [15], a dual band filtering antenna using substrate integrated waveguide (SIW) cavity as a resonator with good isolation between the two bands. Besides, the filtering antenna has a size of $90 \times 90$ $\mathrm{mm}^{2}$. In [16], an improved structure of reconfigurable integrated SIW and patch antenna using the varactor diodes is proposed to achieve a tuning range of around $0.2 \mathrm{GHz}$ with overall dimensions of $98.6 \mathrm{~mm} \times 92.6 \mathrm{~mm}$.

In this paper, a dual band filter-antenna with fixed and frequency-agile bands for wireless communications and CR applications is presented and discussed. Section 2 presents the configuration of the filter-antenna. Section 3 discusses the experimental measurements and observations. In conclusion, a summary of the work is summed up. 


\section{Filter-Antenna Design}

This part presents and discusses the design of the filterantenna. Figure 1 displays the 2D layout of the suggested structure. Obviously, the antenna is composed of tunable bandpass filter connected and matched to the radiating patch. The bandpass filter is composed of a folded arms open loop resonator (FAOLR). The FAOLR is loaded with a varactor diode for reconfiguration operation of the tunable band as we did in [17] as a summary of the simulation results of this study. The filter-antenna has dimensions of $40 \mathrm{~mm} \times 40 \mathrm{~mm}$. The strip $W_{2} \times L_{2}$ has dimensions of $12 \mathrm{~mm} \times 2 \mathrm{~mm}$ which is responsible for the impedance matching. The patch dimensions are $W=L=18 \mathrm{~mm}$. The transmission line dimensions are $W_{l}=1.9 \mathrm{~mm}, W_{t}=0.8 \mathrm{~mm}, g_{t}=0.7 \mathrm{~mm}$, and $L_{l}=19 \mathrm{~mm}$. The resonator dimensions are $X_{r}=7.5 \mathrm{~mm}, Y_{r}=$ $4.5 \mathrm{~mm}, C_{r}=0.4 \mathrm{~mm}$, and $L_{r}=0.5 \mathrm{~mm}$. The reduced ground plane has a length of $L_{g}=19 \mathrm{~mm}$ while the slot defected ground structure (DGS) has dimensions of $2.8 \mathrm{~mm} \times 0.6 \mathrm{~mm}$ with $1.5 \mathrm{~mm}$ separation distance between the two etched slots. The etched slots are corresponding to a resonant circuit and considered as a parallel LC circuit. At any frequency lower than the resonant frequency of the slots, the parallel circuit acts as an inductor [18], [19], [20]. Therefore, they improve the coupling and the matching between the feed line and the FAOLR resonator. Furthermore, the dimensions of the DGS does not have any significant effect on the desired dual frequencies of the capacitively loaded FAOLR. A varactor is attached between the FAOLR and the ground to accomplish miniaturization and reconfiguration. The varactor diode is realized with its equivalent circuit model [17], [18].

The reconfiguration operation of the proposed design is achieved through changing the capacitance of the varactor $(C)$ from 2.67 to $0.63 \mathrm{pF}$ which will tune the second resonance of the FOLAR. The antenna is designed to operate at a continuous tuning range of $3.18 \mathrm{GHz}$ and a fixed band of 4.3 $\mathrm{GHz}$, simultaneously. The antenna is designed using the CST microwave studio software using RO3003 substrate with $\varepsilon_{r}=$ 3 and $h=0.762 \mathrm{~mm}$. The simulated $|\mathrm{S} 11|$ of the proposed filter-antenna accomplishes a continuous reconfiguration band of $3.18 \mathrm{GHz}$ from 4.6 to $7.8 \mathrm{GHz}(|\mathrm{S} 11| \leq-10 \mathrm{~dB})$ for a capacitance of 2.25 to $0.16 \mathrm{pF}$ with $52 \%$ frequency tuning range. The desired wideband matching characteristic is achieved through the two rectangular etched slots in the ground plane as explained in [18]. It should be noticed that the reverse voltage increases, the filter-antenna resonates at high frequencies as indicated in Fig. 1(c). For studying the influence of the gap $g t$ on performance of the proposed design, parametric study for different values of the distance $g t$ has been done as shown in Fig. 1(d). It is noticed that the distance $g t$ has a slight effect on the $|\mathrm{S} 11|$ parameters of the proposed filter-antenna as shown in Fig. 1(d). The filterantenna is simulated with and without the SMD capacitor $C$ $=0.6 \mathrm{pF}$ as it will be tested at this value at the measurement as shown in Fig. 2. Noticeably, the attached capacitor miniaturizes the second resonant frequency from $9.5 \mathrm{GHz}$ to $6 \mathrm{GHz}$ which achieves the compactness of the design.

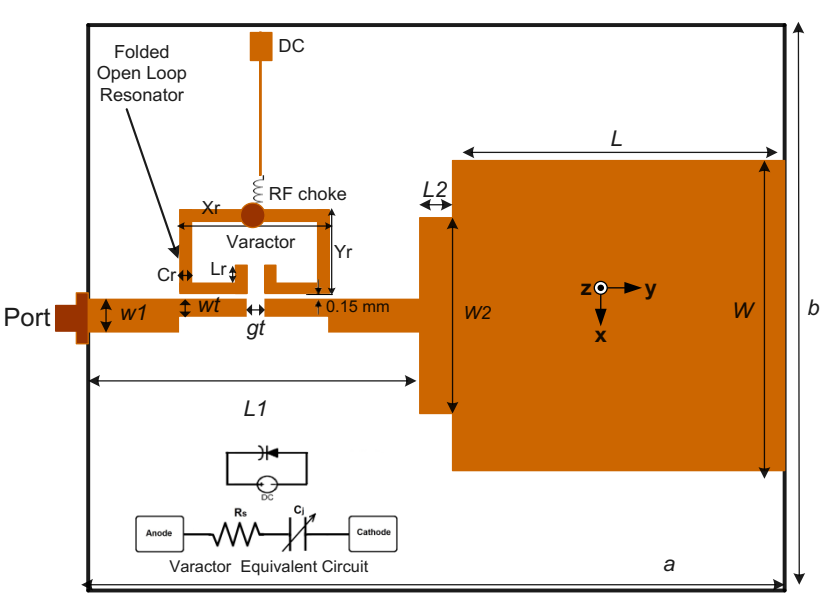

(a)

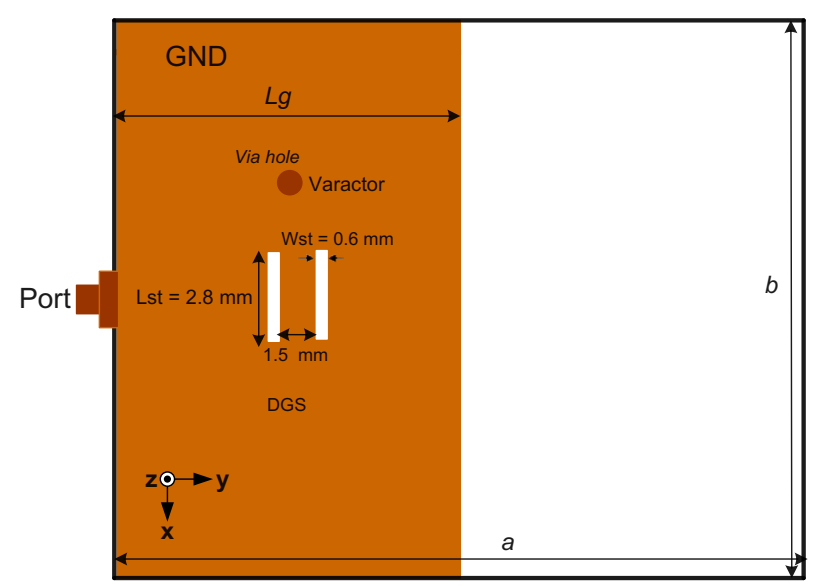

(b)

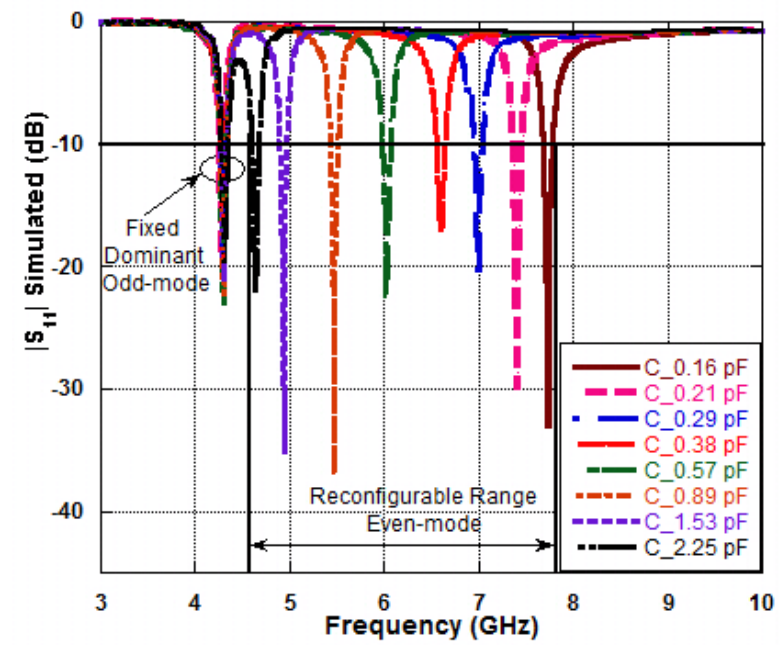

(c) 


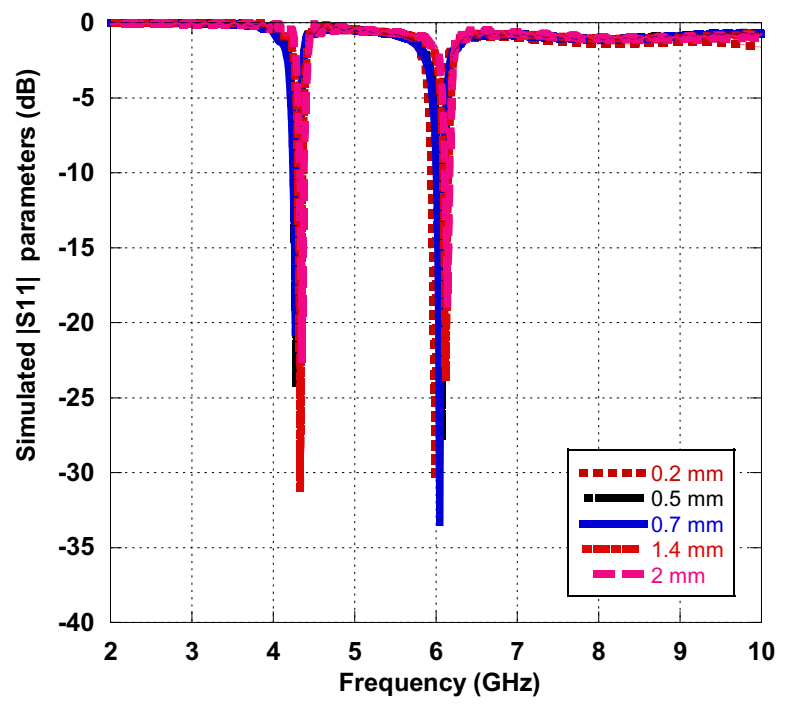

(d)

Figure 1: Geometry of the proposed dual band filterantenna: (a) Top view. (b) Bottom view. (c) Simulated $|\mathrm{S} 11|$. (d) Parametric study of the distance $g t$.

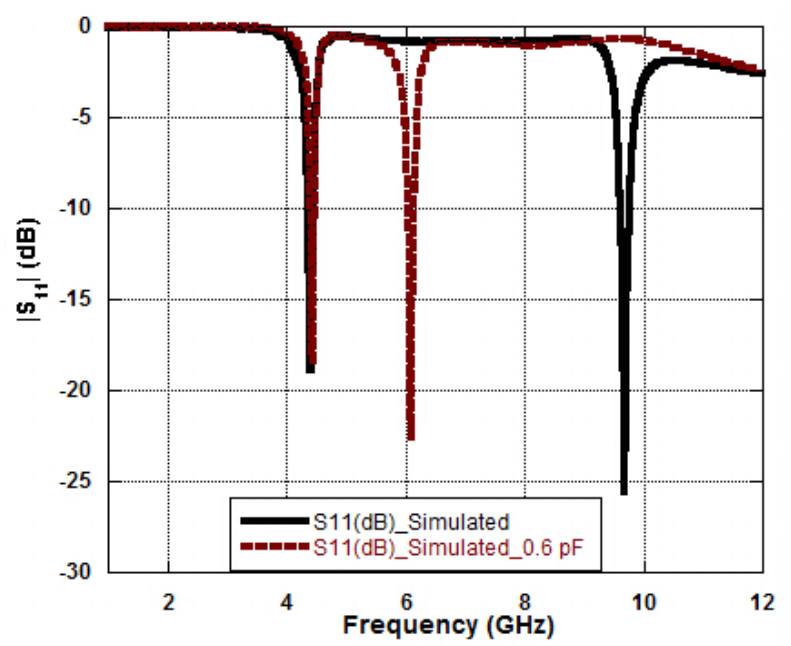

(d)

Figure 2: Simulated $|\mathrm{S} 11|$ with and without $C=0.6 \mathrm{pF}$.

\section{Experimental Measurements and Discussions}

The suggested design is fabricated and tested at one capacitance for confirmation. The measurements are conducted using N5222A PNA microwave network analyzer with a maximum frequency range of $26.5 \mathrm{GHz}$. The pictures of the fabricated sample are shown in Fig. 3. A prototype of the filter-antenna is fabricated and measured at one selected capacitance of $0.6 \mathrm{pF}$ to confirm the simulation results. Moreover, the filter-antenna is tested and measured without the capacitor. All measured results are compared with the simulated results as shown in Fig. 3.

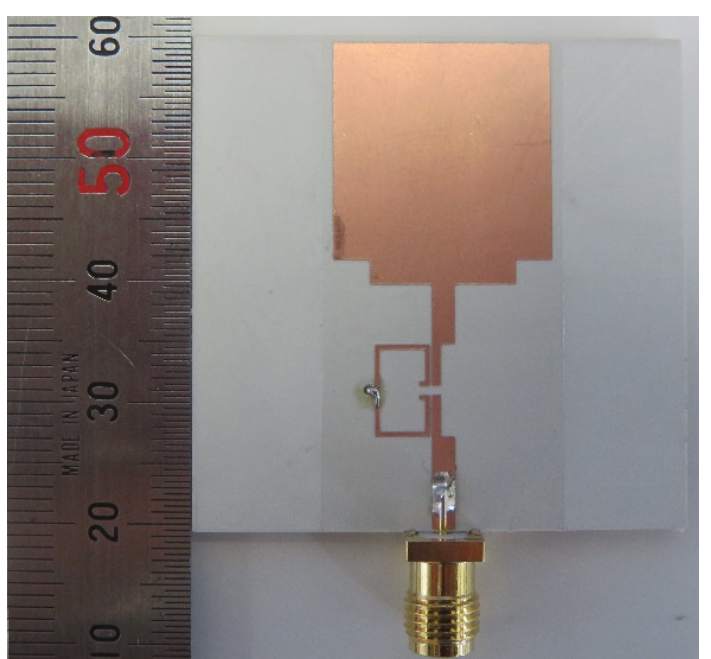

(a)

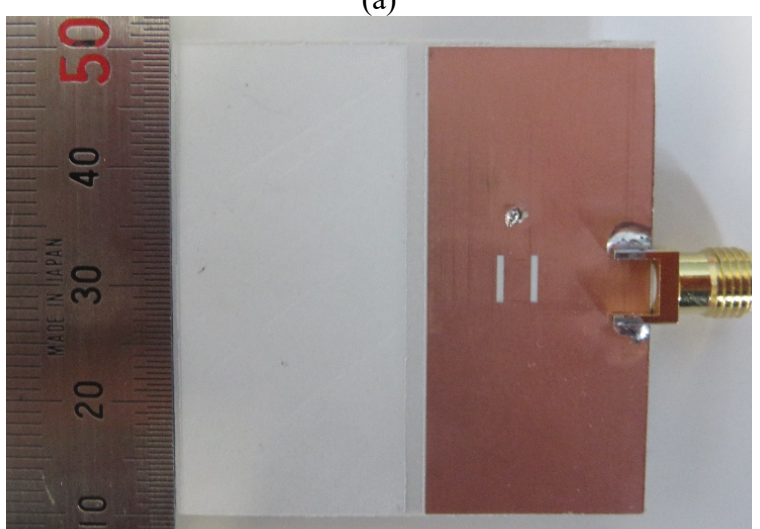

(b)

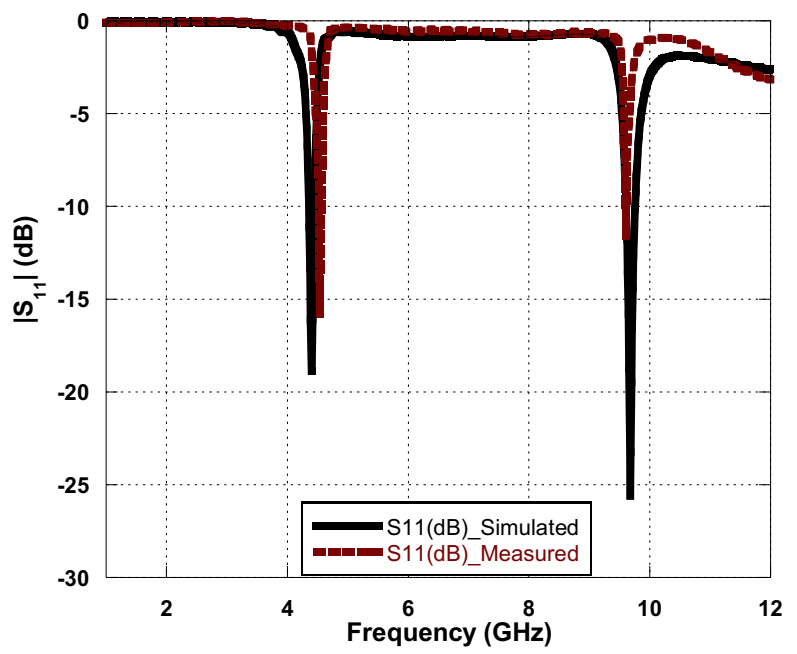

(c) 


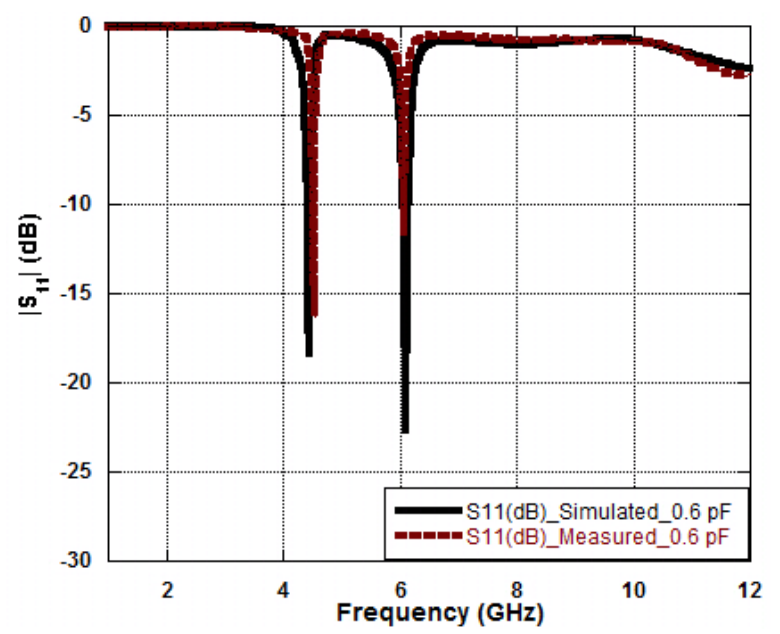

(d)

Figure 3: Photos of the prototype and results: (a) Upper view. (b) Bottom view. (c) $|\mathrm{S} 11|$. (d) $|\mathrm{S} 11|$ with and without $\mathrm{C}=0.6 \mathrm{pF}$.

Good agreement between the measured and the simulated $|\mathrm{S} 11|$ of the proposed filter-antenna is observed as shown in Fig. 3. As a result, these outcomes confirm that the antenna is appropriate for fixed and frequency-agile bands communication applications. Moreover, the simulation and measurement data of the realized gains are plotted and they are in acceptable agreements as shown in Fig. 4. Figure 4 indicates that the antenna has two peaks at the two operating frequencies of 4.3 and $6 \mathrm{GHz}$. This confirms the proper operation of the proposed filtering antenna at both working bands. As well, it has low gains at the non-operating bands.

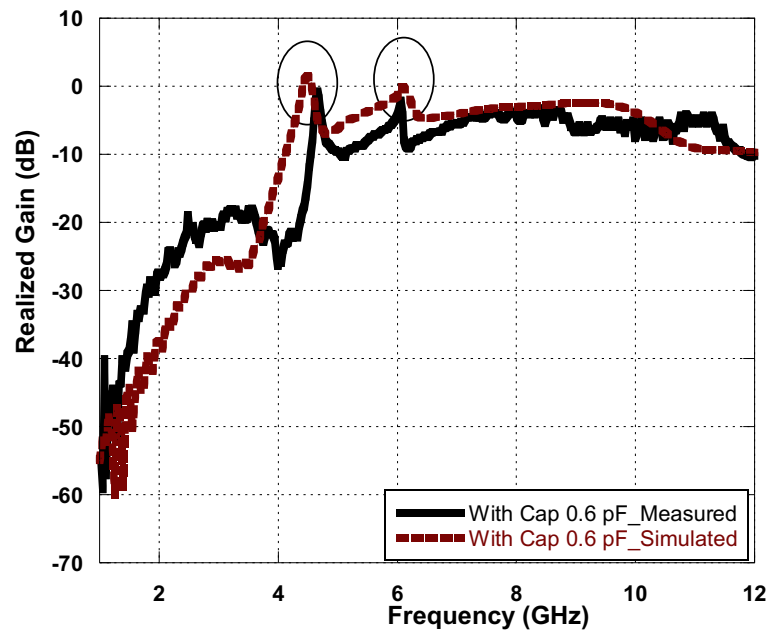

Figure 4. The simulated and measured realized gains.

For more confirmation, the normalized radiation patterns in the $\mathrm{E}$ and $\mathrm{H}$ planes at the selected capacitance $(C=0.6 \mathrm{pF})$ for the operating frequencies $(3.4$ and $6 \mathrm{GHz})$ are plotted in Fig. 5. Furthermore, the measured patterns are compared to the simulated ones. The filter-antenna exhibits stable omnidirectional radiation patterns at the two frequencies. However, some minor deviations are observed in the E plane at $6 \mathrm{GHz}$ which is due to the soldering effects of the capacitance and the coaxial cable.

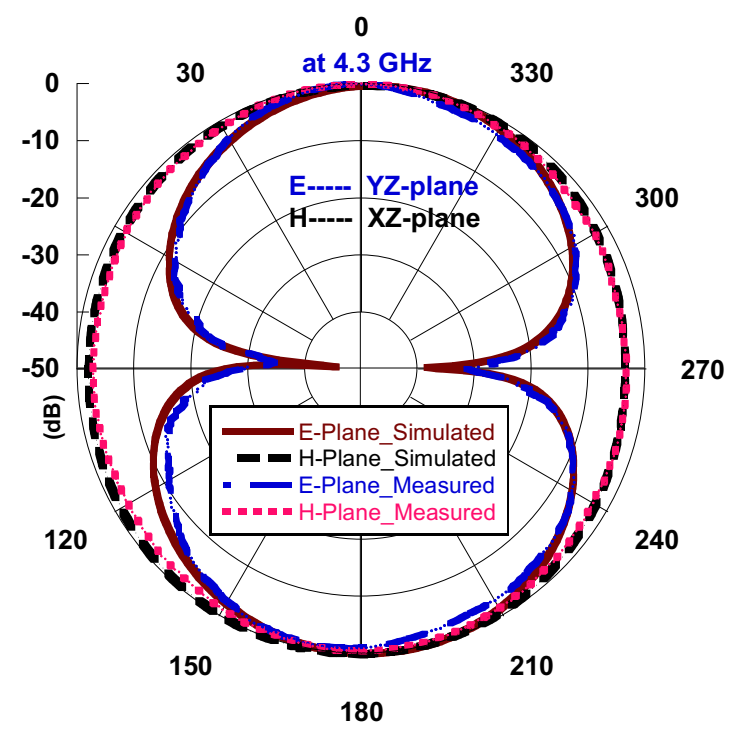

(a)

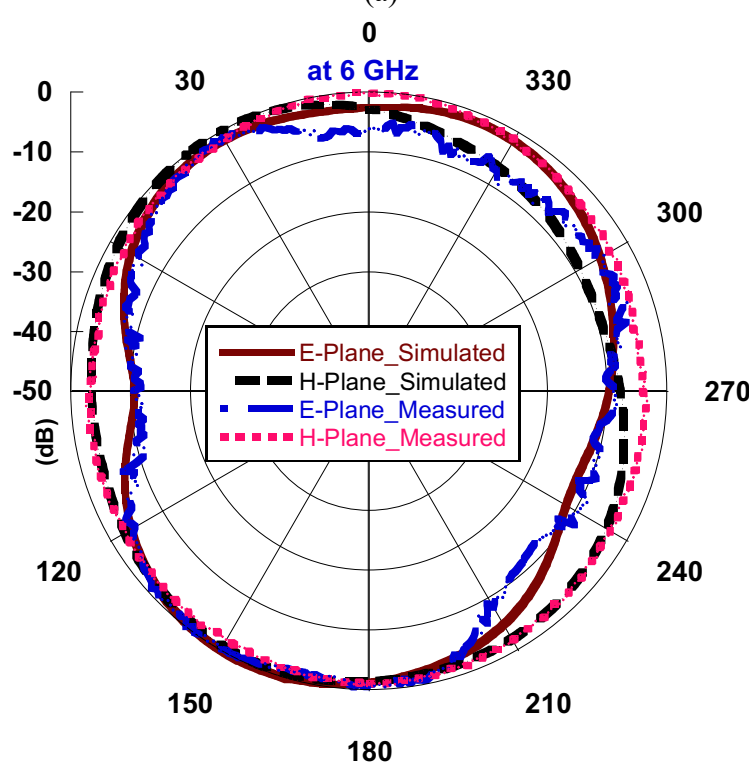

(b)

Figure 5. The radiation patterns of the prototype at $\mathrm{C}=$ $0.6 \mathrm{pF}$. (a) dominant-mode at $4.3 \mathrm{GHz}$. (b) even-mode at $6 \mathrm{GHz}$.

\section{Conclusions}

In this paper, the experimental work of a low-profile filterantenna with a fixed band and a reconfigurable frequency band for wireless communications and CR applications is presented and discussed. The filter-antenna is realized by attaching a tunable bandpass filter to the feed line of a UWB antenna. A wide reconfigurable band of $3.18 \mathrm{GHz}$ from 4.6 to $7.8 \mathrm{GHz}$ is effectively achieved by changing the capacitance of the varactor diode. Furthermore, the filter antenna is tested at $0.6 \mathrm{pF}$ for validation. The radiation patterns are steady at the dual band of operation at 4.3 and 6 GHz. The agreement between the simulated and measured data confirms the operation of the proposed design. 


\section{References}

[1] Jawad Y. Siddiqui, Chinmoy Saha, and Yahia M. M. Antar, "A novel ultra Wideband (UWB) printed antenna with a dual complementary characteristic," IEEE Antennas and Wireless Propagation Letters, vol. 14, pp. 974-977, 2015.

[2] Kumud R. Jha, Bisma Bukhari, Chitra Singh, Ghanshyam Mishra, and Satish K. Sharma, "Compact planar multi-standard MIMO antenna for IoT applications," IEEE Transactions on Antennas and Propagation, vol. 66, no. 7, pp. 3327-3336, 2018.

[3] M. F. Habash, A. S. Tantawy, Hany A. Atallah, and A. B. Abdel-Rahman, "Compact size triple notched-bands UWB antenna with sharp band-rejection characteristics at WiMAX and WLAN bands," Advanced Electromagnetics (AEM), vol. 7, no. 3, pp. 99-103, August 2018.

[4] L. A. Shaik, C. Saha, Y. M. M. Antar, and J. Y. Siddiqui, "An antenna advance for cognitive radio: introducing a multilayered split ring resonator-loaded printed ultrawideband antenna with multifunctional characteristics," IEEE Antennas and Propagation Magazine, vol. 60, no. 2, pp. 20-33, April 2018.

[5] S. Kingsly, D. Thangarasu, M. Kanagasabai, M. Gulam Nabi Alsath, T. Rama Rao, P. Sandeep Kumar, Y. Panneer Selvam, S. Subbaraj, and P. Sambandam, "Multiband reconfigurable filtering monopole antenna for cognitive radio applications," IEEE Antennas Wireless Propagation Letters, vol. 17, no. 8, pp. 14161420, 2018.

[6] R. Hussain and M. S. Sharawi, "A cognitive radio reconfigurable MIMO and sensing antenna system," IEEE Antennas and Wireless Propagation Letters, vol. 14, pp. 257-260, 2015.

[7] J. Costantine, Y. Tawk, S. E. Barbin, and C. G. Christodoulou, "Reconfigurable antennas: design and applications," Proceedings of the IEEE, vol. 103, no. 3, pp. 424-437, March 2015.

[8] Eiyong Park, Daecheon Lim, and Sungjoon Lim, "Dualband band-Pass filter with fixed low band and fluidicallytunable high band," sensors (basel), vol. 17, no. 8, Aug. 2017.

[9] N. Behdad and K. Sarabandi, "Dual-band reconfigurable antenna with a very wide tunability range," IEEE Transactions on Antennas and Propagation, vol. 54, no. 2, pp. 409-416, Feb. 2006

[10] M. B. Kakhki and P. Rezaei, "Reconfigurable microstrip slot antenna with DGS for UWB applications," International Journal of Microwave and Wireless Technologies, vol. 9, no. 7, pp. 1517-1522, 2017.

[11]S. Sah, M. R. Tripathy, and A. Mittal, "Multiband and miniaturized dual layer antenna incorporated with FSS and DGS," Advanced Electromagnetics (AEM), vol. 7, no. 1, pp. 1-6, Jan 2018.
[12] Y. Tawk, A. El-Amine, S. Saab, J. Costantine, F. Ayoub, and C. G. Christodoulou, "A software-defined frequency-reconfigurable meandered printed monopole," IEEE Antennas and Wireless Propagation Letters, vol. 17, no. 2, pp. 327-330, 2018.

[13] C. Saha, L. A. Shaik, R. Muntha, Y. M. M. Antar and J. Y. Siddiqui, "A dual reconfigurable printed antenna: design concept and experimental realization," IEEE Antennas and Propagation Magazine, vol. 60, no. 3, pp. 66-74, June 2018.

[14]H. F. Abutarboush, R. Nilavalan, S. W. Cheung, and K. M. Nasr, "Compact printed multiband antenna with independent setting suitable for fixed and reconfigurable wireless communication systems," IEEE Transactions on Antennas and Propagation, vol. 60, no. 8, pp. $3867-$ 3874, Aug. 2012.

[15] K. Dhwaj, H. Tian, and T. Itoh, "Low-profile dual-band filtering antenna using common planar cavity," IEEE Antennas and Wireless Propagation Letters, vol. 17, no. 6, pp. 1081-1084, June 2018.

[16] W. Y. Sam, Z. Zakaria, and H. Lago, "Enhanced design of reconfigurable integrated filter and antenna based on multilayer approach," 18th International Symposium on Antenna Technology and Applied Electromagnetics (ANTEM), Waterloo, 2018, pp. 1-5.

[17]H. A. Atallah, A. B. Abdel-Rahman, K. Yoshitomi, and R. K. Pokharel, "Compact frequency tunable filtenna with wide continuous tuning range using capacitively loaded folded arms open loop resonator for interweave cognitive radio applications," $201633 \mathrm{rd}$ National Radio Science Conference (NRSC), Aswan, 2016, pp. 87-93.

[18] A. B. Abdel-Rahman, A. K. Verma, A. Boutejdar, and A. S. Omar, "Control of bandstop response of Hi-Lo microstrip lowpass filter using slot in ground plane," IEEE Transactions on Microwave Theory and Techniques, vol. 53, no. 3, pp. 10081013, March 2004.

[19] J.-S. Park, J.-S. Yun, and D. Ahn, “A design of the novel coupled-line bandpass filter using defected ground structure with wide stopband performance," IEEE Transactions on Microwave Theory and Techniques, vol. 50, no. 9, pp. 20372043, Sep 2002.

[20]H. A. Atallah, A. B. Abdel-Rahman, K. Yoshitomi, and R. K. Pokharel, "Compact frequency reconfigurable filtennas using varactor loaded T-shaped and H-shaped resonators for cognitive radio applications," IET Microwaves, Antennas \& Propagation, vol. 10, no. 9, pp. 991-1001, 2016. 